\title{
DESIGN OF A FAST GLOBAL ORBIT FEEDBACK SYSTEM FOR THE ADVANCED LIGHT SOURCE*
}

\author{
C. Steier, A. Biocca, E. Domning, S. Jacobson, \\ G. Portmann, Y. Wu, LBNL, Berkeley, CA94720, USA
}

\begin{abstract}
The fast stability of the closed orbit of the electron beam at the Advanced Light Source (ALS) at Lawrence Berkeley National Laboratory fulfills user requirements so far without any fast active correction system. In the range between 0.1 and $500 \mathrm{~Hz}$ the integrated rms closed orbit motion is significantly below one tenth of one sigma beamsizes. For the future there is some user demand to improve this stability further. Moreover, the expansion of the capabilities of the ALS creates new sources of closed orbit noise. Therefore the design of a fast, global orbit feedback system has been started in conjunction with a general upgrade of the ALS control system. It will initially operate with an update rate of $500 \mathrm{~Hz}-1 \mathrm{kHz}$, will include 24 beam position monitors and corrector magnets in each plane and will use standard computer and networking architecture. The system design, measurements of transfer functions and tests with small prototype systems will be presented.
\end{abstract}

\section{MOTIVATION}

The ALS is a third generation synchrotron light source [1] and has been in operation since 1993. Employing a combination of good passive measures and careful engineering of noise sources like power supplies and the cooling system, the short term closed orbit stability in the ALS fulfills the current user requirements. In the range between 0.1 and $500 \mathrm{~Hz}$ the integrated closed orbit motion in the insertion device straights is below $2 \mu \mathrm{m}$ in the vertical plane and about $3 \mu \mathrm{m}$ in the horizontal plane (one sigma beam sizes at $1.9 \mathrm{GeV}$ at that position are about $30 \mu \mathrm{m}$ vertical and $250 \mu \mathrm{m}$ horizontal). Therefore, earlier designs of fast feedback systems at the ALS [2] have not been implemented on the accelerator.

To reduce the impact of long term orbit drifts due to thermal effects and motion on intermediate time scales due to insertion device scans, a combination of a slow, global orbit feedback system ( $1 \mathrm{~Hz}$ update rate) and feed forward systems for each insertion device $(10-200 \mathrm{~Hz}$ update rate) are used [3]. This yields a long term orbit stability (over time scans of a week) of about 10-20 $\mu \mathrm{m}$, dominated by thermally induced drifts in the location of the beam position monitors. Figure 1 shows typical power spectral densities of the fast closed orbit motion at the ALS. The noise at frequencies up to $5 \mathrm{~Hz}$ is caused by power supplies, as well as the spike at $60 \mathrm{~Hz}$, which currently dominates the ver-

\footnotetext{
${ }^{*}$ This work was supported by the U.S. Department of Energy, under Contract No. DE-AC03-76SF00098.
}

tical orbit motion. The noise between about 7 and $30 \mathrm{~Hz}$ is coming from vibration modes of the girders. The BPM electronics used for this measurement which will also be used for the feedback system is a multiplexed BPM design. The multiplexing frequency is high enough for the planned update rate of the feedback system.
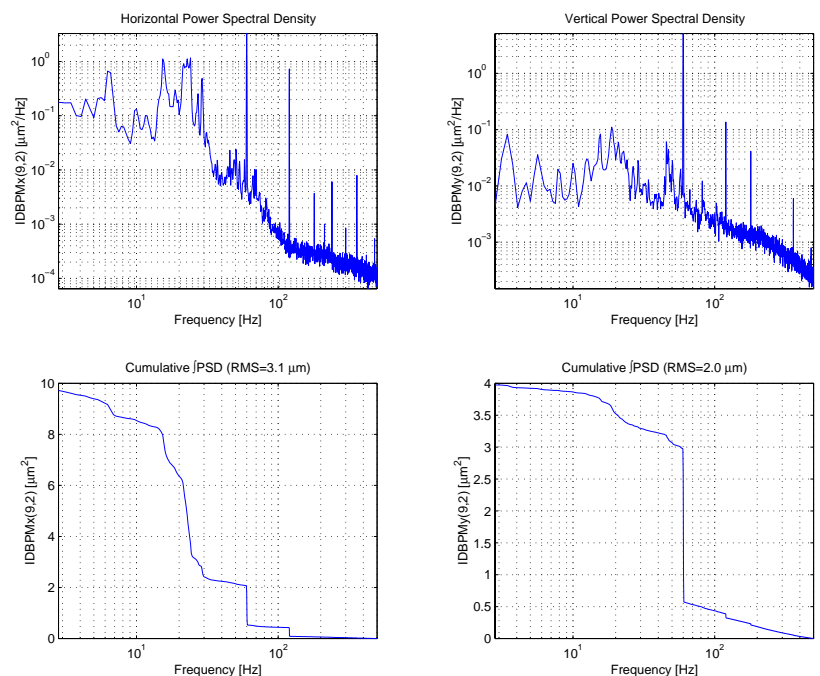

Figure 1: Power spectral density of the closed orbit oscillations in the ALS without orbit feedback.

Since recently, there is user demand to improve especially the short term orbit stability further. This demand is for example driven by the progress in dichroism experiments, which are capable of detecting smaller and smaller asymmetries. Vertical orbit stability (and therefore photon energy stability) is especially crucial for microscopy experiments trying to measure small dichroisms, since a correction of the measurement results for the incoming flux and photon energy is extremely difficult.

Furthermore, there is a constant expansion of the ALS, often creating new sources of closed orbit noise. Elliptically Polarizing Undulators [4] for example require fast focusing compensation, to minimize their influence on the beamsize, which in turn creates fast distortions of the orbit. Other noise sources are active tune/chromaticity compensation schemes, the cryogenics of superconducting magnets or beamlines, etc. To prevent a deterioration of the current orbit stability due to those upgrade projects and ultimately provide a short term submicron orbit stability to enable advanced experiments the design of a fast, global orbit feedback system has been started. The initial goal for 2001 is to operate with an update rate of $500 \mathrm{~Hz}-$ $1 \mathrm{kHz}$. Those parameters are conservative compared to al- 
ready working systems at the APS [5] and NSLS.

\section{FEEDBACK SYSTEM LAYOUT}

With the advent of higher performance networking it appears practical to use it directly for medium performance distributed control systems. In the past higher-cost specialized solutions were often required such as reflective memory. The requirements for this feedback system appear to be compatible with $100 \mathrm{Mbit} / \mathrm{s}$ networking and standard processors.

\subsection{System Configuration}

The system consists of 12 Compact PCI chassis distributed around the ring on a (new,) private, switched $100 \mathrm{Mbit} / \mathrm{s}$ network. Each chassis handles four BPM inputs and four corrector magnet outputs via analog to digital converter (ADC) and digital to analog converter (DAC) boards. There are additional channels available in the crate that later will be used for the (slow) control system interfacing of all powersupplies of the ALS. The subsystems were designed for compatibility with existing ALS controls hardware (e.g. cabling) and are also used for other upgrades and expansion. The CPUs are Motorola PowerPC based $367 \mathrm{MHz}$ boards running Wind River Systems VxWorks/Tornado.

Each crate has a timing board to provide the interrupt synchronizing the inputs and outputs of the control algorithm. The initial plan is to use network packets to synchronize these timers. If necessary, there is a backup plan to distribute a precision timing signal to the cards via hardware. Synchronizing the timers with network packets is planned using a long time constant software based phase locked loop to adjust the period of the timer based on input from a network broadcast packet. Several schemes have been considered but no testing has been attempted thus far.

The ALS control system so far utilizes 16 bit DACs and ADCs. This does not provide a small enough step size for a fast feedback system. Therefore, we are adding a summing junction and using two DAC channels, one for slow control of the magnet setpoint, and the other for fast feedback with a 10:1 attenuator.

\subsection{Algorithms}

A number of slightly different software schemes are being investigated. The process is interrupt driven with the interrupts generated by a timer board. After the interrupt the magnet currents calculated in the last cycle are set, the BPMs are read, the readings are shared via network packets, and the array computations to determine the next outputs are performed. All 12 processors are running in parallel, and each has only $1 / 12$ th of the computations to complete. The most time consuming operation is the sharing of the data via the network. In addition there is a slow loop running, which synchronizes the timer boards. The opti- mization of the parameters of the controller which computes the new setpoints is described in section 4.

Predictions and measurements of I/O, network, and processing time indicate that the whole process will take approximately half the $1 \mathrm{~ms}$ time window, which is available at an update rate of $1 \mathrm{kHz}$.

\subsection{Networking Issues}

All 12 systems need all BPM values to perform their control calculations. To share this data UDP broadcasts from all nodes are used. These broadcasts are sent at about the same time by all 12 systems but they do not collide on the network since a full duplex switch is employed. All systems can in fact transmit simultaneously in which case the first packet coming into the switch is transmitted by the switch to all outputs at once, and the other 11 packets are queued in the switch memory. They then go out with a minimum gap between them. This process requires about one third of the total time budget for an update cycle. The feedback software checks to see if all BPM values are available for the current cycle, and if they are not that cycle is skipped and counted so the feedback system degrades gracefully in the presence of other high network traffic loads. The network is separate from other controls to reduce the potential for this event.

\section{PERFORMANCE TESTS}

Two timing tests were run to determine whether the planned CPU and network configuration could accommodate the timing requirements. The first test performed the matrix operations using arrays of random data. The test calculated the dot products of four arrays of 36 doubles with coefficient arrays of the same size. Each run of the test therefore consisted of 144 double-size floating point multiplications and 144 sums. On a 367 MHz Power PC (Motorola MCP750) this test ran in an average time of $2 \mu \mathrm{s}$. The second test simulated the network and CPU load using two MCP750s connected to each other through a $100 \mathrm{Mbit}$ Ethernet switch. One CPU sent a 36-byte datagram packet to the second; the second then sent 11 packets of the same type and size back to the first. This test averaged $300 \mu \mathrm{s}$ per 12-packet transfer. Even though this test only used two CPUs, it is expected that the time needed for the network communication in the real architecture with 12 crates will be very similar, due to the use of the switch and the 12 individual, full-duplex connections. Tests with all 12 CPUs will be carried out soon.

\section{CONTROLLER DESIGN}

The controller which is used to compute the new output setpoints in each feedback system step is crucial for the overall performance of the feedback system. In order to optimize it (and especially its parameters), it is essential to have a good model of the complete feedback system. 


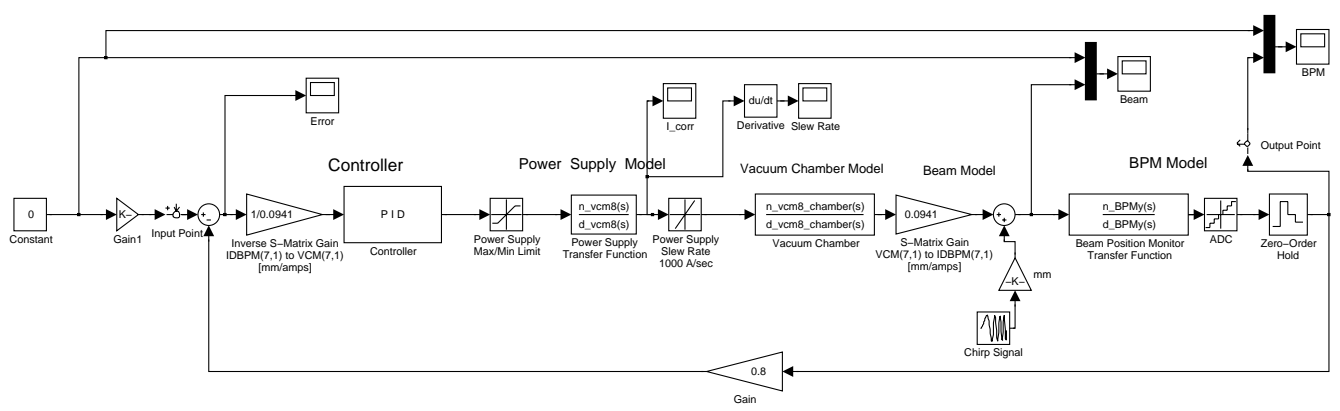

Figure 2: One of the Matlab-Simulink models used to optimize the performance of the smallest building block of the feedback system: One BPM, one ADC and DAC, the controller and one corrector magnet.

Therefore, a set of transfer function measurements (measuring current, magnetic fields or beam motion both on tests stands and on the accelerator) was carried out, charaterizing all important elements involved (i.e. power supplies, magnets, vacuum chambers, beam, BPMs). Examples of measured transfer functions are shown in figure 3. The figure shows the transfer functions of a corrector power supply (for two different driving amplitudes) and the combined transfer function of power supply, magnet, vacuum chamber, beam and BPM.

For the simulations to optimize the controller, all transfer functions were fitted and some non-linearities (e.g. slew rate limits of power supplies) were included as well.

The simulations are performed in Matlab, using the Simulink toolbox. Figure 2 shows an example of one of the models used to optimize the system. This specific model consists of the smallest building block of the system (just one BPM and one corrector magnet). Early results
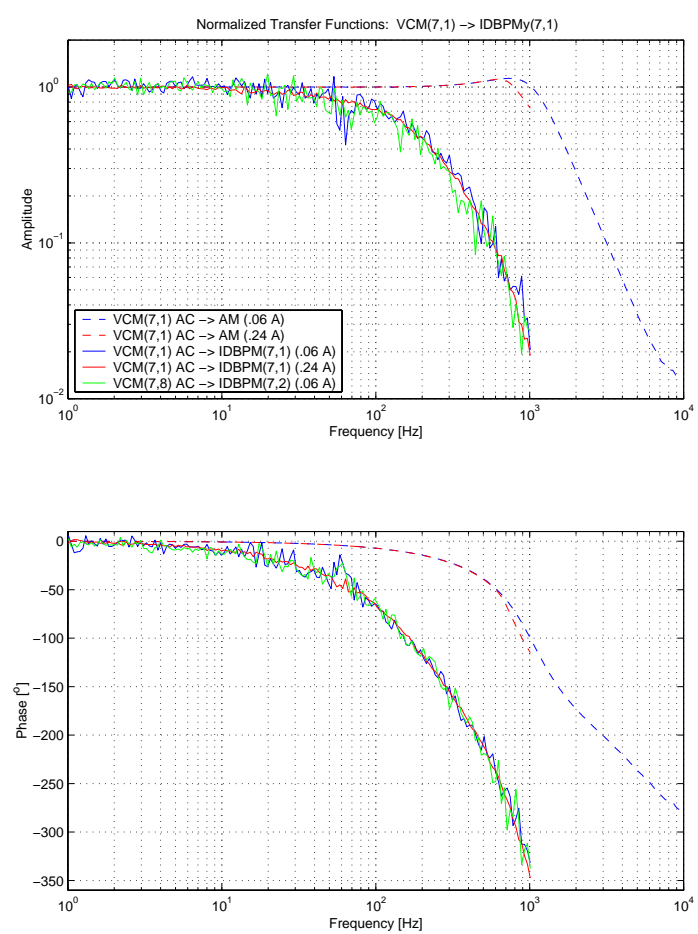

Figure 3: Transfer function of an ALS corrector magnet power supply and combined transfer function of power supply, magnet, vacuum chamber and beam. of the simulations indicate that the hardware limitations (bandwidth of powersupplies, magnets, vacuum chamber and BPMs) are at high enough frequencies, allowing good noise suppression in the desired frequency range (up to $100 \mathrm{~Hz}$ ). Limitations due to the finite ADC resolution, the sample rate (initially $500 \mathrm{~Hz}-1 \mathrm{kHz}$, later with improvements in networking technology higher), parallelization have to be studied in more detail, and a systematic optimization of the controller has to be performed.

\section{SUMMARY AND OUTLOOK}

A design for a fast orbit feedback system for the ALS was completed. Its purpose is to maintain the current, good short term stability of the closed orbit and ultimately improve it to submicron stability for future experiments. All hardware components have been designed and ordered and the installation is planned for this fall. Several performance tests have been carried out and the software development is well on the way. To prepare the optimization of the controller, transfer function measurements were performed, enabling the creation of Simulink models to study the system. The software development and optimization will continue and the commissioning of the system will start at the end of this year. For the future, network upgrades are planned to increase the update rate, as well as improvements of individual components.

\section{REFERENCES}

[1] 1-2 GeV Synchrotron Radiation Source Conceptual Design Report, LBNL publication PUB-5172 Rev. (1986)

[2] G. Portmann, J. Bengtsson, A closed-loop photon beam control study for the Advanced Light Source, Proceedings of PAC 1993, Washington D.C.

[3] G. Portmann, Slow orbit feedback at the Advanced Light Source using Matlab, Proceedings of PAC 1999, New York, NY

[4] C. Steier, Commissioning of the first elliptically polarizing undulator at the ALS, Proceedings of EPAC2000, p. 2343, Vienna, Austria

[5] J. Carwardine, et al., Commissioning of the APS real-time orbit feedback system, Proceedings of PAC 1997, p. 2281, Vancouver, BC 